\title{
EL EMBARAZO COMO SITUACIÓN DE VULNERABILIDAD ANTE LA VIOLENCIA \\ DE GÉNERO: PERSPECTIVA DE LAS MATRONAS DE ATENCIÓN PRIMARIA SOBRE SU CAPACITACIÓN EN DETECCIÓN Y ABORDAJE ${ }^{1}$
}

Pregnancy as a Situation of Vulnerability to Gender-Based Violence: The Perspective of Primary Care Midwives on Their Training in Detection and Response to IPV

María Analía Gómez-Fernández, ${ }^{1}$ Montserrat Payà-Sánchez, ${ }^{2}$ Mónica Isidro-Albaladejo, ${ }^{3}$ María García-del Arco, ${ }^{4}$ Raquel Molina-Ordoñez, ${ }^{4}$ Pilar Cabrera-García ${ }^{4}$

Correo electrónico: analiagomez2@yahoo.es

1.- Atención Primaria, Sector Zaragoza III. Instituto Universitario de Investigación en Atención Primaria (IDIAP Jordi Gol) (Barcelona, España).

2.- Departamento de Teoría e Historia de la Educación. Instituto de Investigación en Educación. Facultad de Educación. GREM: Grupo de investigación en Educación Moral. Universitat de Barcelona (Barcelona, España).

3.- Facultad de Medicina y Ciencias de la Salud. Universitat de Barcelona. Atención a la Salud Sexual y Reproductiva (ASSIR) Baix Llobregat Centre. Institut Català de la Salut. Instituto Universitario de Investigación en Atención Primaria (IDIAP Jordi Gol) (Barcelona, España).

4.- Atención a la Salud Sexual y Reproductiva (ASSIR) Delta de Llobregat. Institut Català de la Salut. Instituto Universitario de Investigación en Atención Primaria (IDIAP Jordi Gol) (Barcelona, España).

Recibido: 23/02/2020 Aceptado: 20/04/2020

${ }^{1}$ La investigación que ha dado lugar a estos resultados ha sido financiada por el proyecto Recercaixa (2016ACUP 0083). Y financiada parcialmente por la Fundación Enfermería y Sociedad en el marco de las Ayudas a la Investigación Enfermera (PR-050/16). Este trabajo deriva de la Tesis doctoral de la Primera Autora. Resultados preliminares del trabajo fueron presentados como comunicación tipo poster en el XVIII Congreso de la Federación de Asociaciones de Matronas de España, celebrado del 06 al 08 de junio de 2019 en Málaga.
} 


\section{RESUMEN}

INTRODUCCIÓN. Las matronas están en una posición privilegiada para identificar y abordar la violencia de género, pero para ello necesitan la formación adecuada. OBJETIVo. El objetivo de este trabajo es conocer la formación realizada por las matronas sobre violencia de género, la percepción de su capacitación para detectar y abordar la violencia de género y sus necesidades formativas. METODOLOGÍA. Estudio cualitativo con un enfoque fenomenológico interpretativo. Se hicieron entrevistas en profundidad a doce matronas que trabajan en atención primaria. RESULTADOS. Las matronas tienen formación y conocimientos sobre violencia de género, obtenidos mayoritariamente gracias a la formación continuada y su experiencia profesional. Sin embargo, esta formación no es suficiente para sentirse capacitadas para la detección y abordaje de la violencia de género. CONCLUSIONES. Las matronas tienen interés por mejorar su formación y capacitación en cuanto a la detección y abordaje de la VG. Sería deseable disponer de un programa de formación continuada en violencia de género para las matronas con un carácter flexible y polivalente para adaptarse mejor a sus intereses formativos.

Palabras clave: violencia contra la mujer; embarazo; matrona; formación; investigación cualitativa.

\section{ABSTRACT}

BACKGROUND. Midwives are in a privileged position to identify and help women who suffer from gender violence. However, suitable training is required. AIM. The aim of this article is to identify the training they have received on IPV, how they perceive their skills for detecting and addressing IPV, and to determine their training needs regarding IPV. METHOD. Qualitative methodology with an interpretative phenomenological approach. In-depth interviews were conducted with twelve primary care midwives. RESULTS. The midwives have IPV training and knowledge obtained in most cases through continuous learning and through years of clinical experience. However, it has not been enough for these midwives to feel equipped to adequately detect and address IPV. CONCLUSIONS. The midwives would like to improve their training and skills to better detect and address IPV. It would be ideal to have a continuous training program on IPV for midwives that is flexible and varied enough to fit their training interests.

Keywords: Violence Against Women; Pregnancy; Midwives; Training; Qualitative Research. 


\section{INTRODUCCIÓN}

La violencia de género (VG) es definida por las Naciones Unidas en 1993 como «todo acto de violencia basado en la pertenencia al sexo femenino que tenga o pueda tener como resultado un daño o sufrimiento físico, sexual o psicológico para la mujer, así como las amenazas de tales actos, la coacción o la privación arbitraria de la libertad, tanto si se producen en la vida pública como en la vida privada». La VG tiene una elevada prevalencia: según la Organización Mundial de la Salud (OMS), a nivel mundial, el 30\% de las mujeres que han estado en una relación pareja han experimentado alguna forma de violencia (OMS, 2017). En España, una encuesta a 10171 mujeres determinó que el 12,5\% habían sufrido VG en algún momento de su vida (Delegación del Gobierno para la Violencia de Género, 2015). Durante el embarazo, según un metaanalisis, el 28,4\% de las gestantes sufren violencia psicológica, el 13,8\% física y el 8,0\% abuso sexual (James, Brody, y Hamilton, 2013). La OMS (2013) define la VG como un problema de salud pública porque afecta a la salud física de las mujeres (a causa de las lesiones directas o por somatización) y a su salud mental (provocando depresión, ansiedad, entre otros). La VG es especialmente preocupante durante el embarazo puesto que pude provocar aborto, parto prematuro o incluso muerte neonatal (OMS, 2011).

Las matronas de atención primaria (AP) son el primer punto de contacto de las mujeres embarazadas con el Sistema Nacional de Salud español (SNS); en Cataluña son el profesional de referencia del seguimiento y control del embarazo normal (Generalitat de Cataluña, 2007). En el 2009, el SNS incluyó como competencia de las matronas «detectar situaciones de VG, atendiendo a las necesidades que presenten las mujeres en esta situación» (Ministerio de Sanidad y Política Social, 2009), pero para ello se necesita una formación adecuada. En ese mismo sentido, las Naciones Unidas señalan que la capacitación en VG es particularmente importante para los profesionales de la salud que trabajan en las áreas de obstetricia, ginecología y salud sexual (2011). Por su parte, la Ley Orgánica 1/2004 de Medidas de Protección Integral contra la Violencia de Género del 2004 determina que todas las disciplinas universitarias sanitarias deben incorporar contenidos para la formación en prevención, detección y apoyo a las víctimas, y que se desarrollarán programas de sensibilización y formación continuada del personal sanitario (Jefatura del Estado, 2004). 
Conocer la capacitación y las necesidades formativas de las matronas asistenciales permitirá diseñar futuros programas de formación continuada sobre detección y abordaje de la VG en el embarazo, integrando la perspectiva práctica (favoreciendo la correspondencia entre la formación y las necesidades e intereses del público al que va dirigida) y la perspectiva teórica (atendiendo a las necesidades formativas y a las metodologías de enseñanza-aprendizaje idóneas según la experiencia del profesorado universitario). Para ello, previamente al trabajo que se presenta, se examinó la presencia de contenidos relacionados con VG y la profundidad con la que se abordan en la Licenciatura en Enfermería y en el programa de especialización de Matrona en Cataluña, que, aunque adecuada, requiere ser ampliada (Gómez-Fernández, Goberna-Tricas, y Payà-Sánchez, 2017).

El objetivo del presente trabajo se centra en conocer a través de las narraciones de las matronas de atención primaria la formación que han realizado sobre VG, así como su percepción en relación a su nivel de capacitación en la detección y abordaje de la VG. Conjuntamente, profundizar en sus necesidades formativas sobre VG.

\section{METODOLOGÍA}

\section{Diseño}

Estudio cualitativo con enfoque fenomenológico interpretativo (Mackey, 2005). Pretendiendo comprender las necesidades de capacitación para la detección de la VG durante el embarazo de las matronas de acuerdo con su experiencia.

\section{Ámbito}

Los dos centros de AP con titularidad pública que prestan asistencia a la salud sexual y reproductiva del municipio de L'Hospitalet de Llobregat (L'H). L'H es un municipio de la provincia de Barcelona (España) con 257000 habitantes. Su población es muy heterogénea, con una gran diversidad cultural: el 18,66\% de la población ha nacido fuera de España (Generalitat de Catalunya, 2017). En ambos centros, trece matronas prestan asistencia a una media de 2100 mujeres embarazadas cada año.

El trabajo se llevó a cabo entre octubre del 2016 y febrero del 2017. 


\section{Recogida de datos}

En un primer momento, se contactó con la responsable de formación de la institución sanitaria para obtener información referida a los cursos y jornadas con contenidos en VG realizados desde la propia institución en los últimos 5 años. Se revisaron los programas formativos, determinando el año en que se llevó a cabo, el número de horas impartidas, los temas tratados y la metodología empleada. Seguidamente, en una reunión de equipo, se presentó el estudio a todas las matronas, explicando sus objetivos y la metodología y solicitándoles su colaboración. A continuación, se concretó vía correo electrónico día y hora para realizar una entrevista individual en profundidad cara a cara con cada matrona. Las entrevistas fueros realizadas por la misma investigadora (la cual pertenece al grupo de matronas de AP) y grabadas digitalmente. La duración promedio fue de ochenta minutos. Se tomaron notas de campo durante el proceso. Se utilizó un guion de preguntas abiertas que actuaron como pregunta estímulo (se recoge en la tabla 1). Las entrevistas fueron realizadas en espacios privados del centro de trabajo de las participantes. No hubo necesidad de repetir ninguna entrevista. La saturación se alcanzó a partir de la décima entrevista, a partir de la cual se siguió con el proceso de categorización.

Tabla 1. Guía temática de las entrevistas

¿En qué año terminó sus estudios de enfermería y en qué año la especialidad de matrona?

¿Cuál ha sido su trayectoria profesional?

Durante su formación reglada ¿recibió formación sobre VG?

¿Ha realizado formación continuada sobre VG?, ¿qué contenidos le han ayudado en su práctica y cuáles ha encontrado a faltar?

En su opinión, ¿está extendida la VG en las gestantes que asiste?, ¿cuáles son los riesgos de la VG durante el embarazo?

¿Cómo detecta y aborda los casos de VG?

En su opinión, ¿¿cuáles son las necesidades formativas de las matronas?

Según su experiencia ¿qué propuestas haría para el diseño de futuros programas formativos? 


\section{Sujetos de estudio}

Se entrevistaron a todas las matronas de AP de L'H. Las características de las entrevistadas pueden observarse en la tabla 2 (identificadas como e1-e12). Todas son mujeres, tienen una relación laboral permanente con la institución y experiencia previa en el ámbito hospitalario.

Tabla 2. Características de las matronas entrevistadas.

\begin{tabular}{|c|c|c|c|c|}
\hline Matrona & Edad & Enfermería ${ }^{a}$ & Matrona ${ }^{b}$ & $\begin{array}{l}\text { Matrona en } \\
\text { atención primariac }\end{array}$ \\
\hline $\mathrm{e} 1$ & 60 & 1977 & 1979 & 15 \\
\hline e2 & 59 & 1978 & 1981 & 17 \\
\hline e3 & 59 & 1978 & 1982 & 26 \\
\hline $\mathrm{e} 4$ & 58 & 1982 & 1984 & 20 \\
\hline $\mathrm{e} 5$ & 56 & 1982 & 1985 & 24 \\
\hline e6 & 56 & 1982 & 1986 & 27 \\
\hline $\mathrm{e} 7$ & 55 & 1983 & 1986 & 30 \\
\hline $\mathrm{e} 8$ & 53 & 1985 & 1987 & 14 \\
\hline $\mathrm{e} 9$ & 53 & 1985 & 1996 & 20 \\
\hline $\mathrm{e} 10$ & 41 & 1996 & 1998 & 13 \\
\hline $\mathrm{e} 11$ & 39 & 1998 & 2013 & 2 \\
\hline $\mathrm{e} 12$ & 34 & 2003 & 2008 & 8 \\
\hline
\end{tabular}

Nota: a año de finalización de los estudios de enfermería; b año de finalización de los estudios de matrona; $\mathbf{c}$ años de experiencia como matrona en atención primaria

\section{Análisis}

Tras la transcripción textual de las grabaciones, los textos fueron enviados vía correo electrónico a las entrevistadas (las cuales hicieron modificaciones mínimas). El análisis de las entrevistas se basó en lo que refirieron las matronas y en el sentido que ellas mismas fueron generando al reflexionar sobre su ejercicio profesional. La información fue analizada con un enfoque inductivo: descubrimiento, codificación de los datos y relativización (Taylor \& Bogdan, 1987). Tres de las investigadoras identificaron de forma independiente los códigos iniciales para posteriormente hacer una puesta en común y consensuar aplicaciones (es de mencionar que el grado de acuerdo fue elevado). Con el apoyo del programa Atlas-Ti v.7.5.10 se procedió a sistematizar la codificación, estableciendo las relaciones entre categorías y subcategorías, como se recoge en la figura 1. Así se obtuvieron tres categorías de códigos y veintitrés subcategorías o unidades de significado. 
Figura 1. Árbol de codificación. Las cuatro categorías de códigos, veintiséis subcategorías y la relación entre ellos

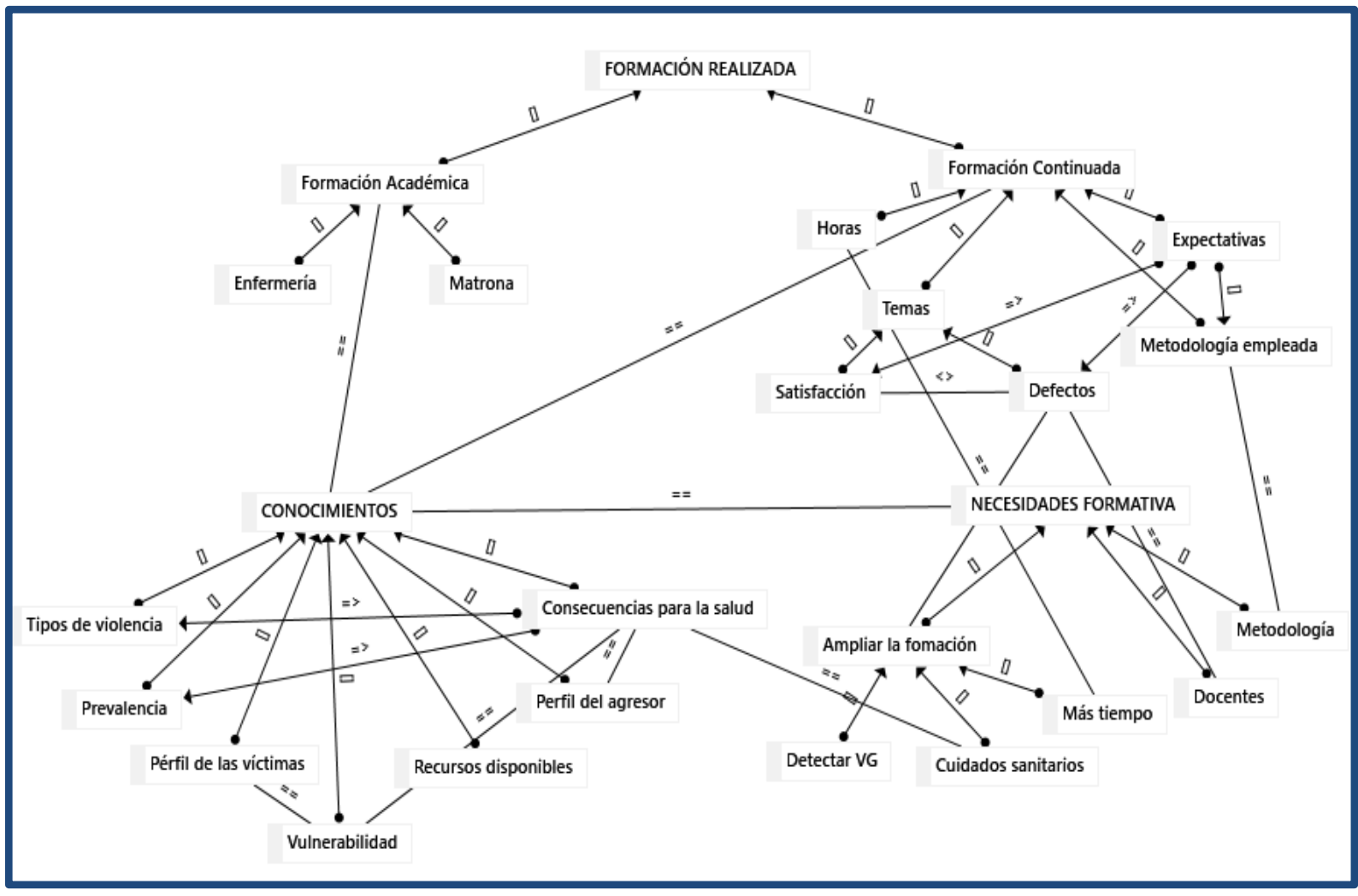

Nota: [] Es parte de; => Es causa de; == Está asociado con

\section{Criterios de rigor de la investigación}

Para garantizar la confiabilidad de la investigación, se tuvo en cuenta los siguientes criterios de rigor: la credibilidad (se pudo entrevistas a todas las matronas de AP que realizan asistencia en L'H); la fiabilidad (las autoras se reunieron en diversas ocasiones para consensuar el análisis de la información y triangular la aplicación de los códigos); la transferibilidad (se ha descrito minuciosamente el proceso de obtención y análisis de datos); y la confirmabilidad (se dispone de las grabaciones, transcripciones, notas de campo, diario de investigación y análisis para su contraste) (Graneheim y Lundman, 2004; Skrtic, 1985).

\section{Aspectos éticos}

Previamente a la realización de la investigación, se obtuvo la aprobación del proyecto por parte de la Comisión de Bioética de la Universidad de Barcelona (IRB00003099) y del Comité Ético de Investigación Clínica del IDIAP Jordi Gol (P15/129). Cuando las matronas aceptaron ser entrevistadas, se les facilitó una hoja informativa sobre la 
investigación y se solicitó su consentimiento por escrito para grabar la entrevista, asegurando el anonimato cuando los datos fueran publicados. Las dudas fueron atendidas por la entrevistadora. Se puso a disposición de las matronas los datos de contacto de las investigadoras.

\section{RESULTADOS}

El análisis de las entrevistas ha proporcionado un denso conocimiento sobre la formación, capacitación y saberes de las matronas, construidos desde su formación y experiencia. También ha posibilitado identificar sus necesidades e intereses formativos sobre VG. Se presenta esta información estructurada a partir de los dos temas identificados como centrales en el análisis (capacitación de las matronas en violencia de género y necesidades formativas) y de las subcategorías más significativas que han permitido integrar en ellas también lo singular.

\section{Capacitación de las matronas en violencia de género}

Distinguiremos en este apartado entre la formación recibida durante los estudios universitarios (formación reglada) y la relativa a formación continuada (FC).

\section{Formación reglada}

De las doce matronas entrevistadas solo una recuerda haber recibido formación sobre VG durante sus estudios de enfermería. Lo hizo gracias a su inscripción voluntaria a un curso de créditos optativos. El resto de ellas no recuerda haber recibir formación sobre VG durante su formación como enfermera. Lo atribuyen principalmente a que se formaron hace años en una época en que la VG era un aspecto que no se abordaba de forma abierta, no era tan visible socialmente como en la actualidad. Un ejemplo lo podemos encontrar en el siguiente caso:

No, la verdad es que no, hace treinta años que estudié enfermería. Este tema ni se hablaba [...] ni en las clases, ni a nivel de profesionales se comentaba. [...] La gente ahora tenemos mucha más consciencia de este problema. (e8)

Durante el periodo de formación para la especialización como matrona, las cuatro que hicieron la residencia en los últimos 21 años (e9, e10, e11 y e12) recibieron formación sobre VG (dos de ellas antes incluso de la promulgación de la mencionada Ley Orgánica 1/2004), aunque reconocen que la formación recibida fue de pocas horas y en aquel 
momento no se sintieron motivadas para profundizar de forma significativa en el tema. Le dieron poca importancia e incluso lo olvidaron, como en el siguiente caso:

Fue una sesión, una tarde creo, pero no recuerdo exactamente los contenidos. (e11)

Las ocho restantes, todas formadas hace más de 30 años, no recuerdan haber recibido formación sobre VG durante el periodo en que cursaron la especialidad:

En absoluto, para nada [...]. A nosotras nos prepararon mucho de cara al parto y al puerperio inmediato, pero ni seguimiento del embarazo, ni lactancia, ni problemas sociales. (e1)

\section{Formación continuada}

Todas las matronas han realizado FC sobre VG. Esta FC fue interna, es decir, organizada por la propia institución sanitaria, impartida dentro del horario laboral y dirigida conjuntamente a diferentes profesionales de la salud (médicos, enfermeros, matronas, auxiliares de enfermería). Según los programas formativos revisados, se realizaron tres cursos con contenido relacionado con la VG: a ellos asistieron todas las matronas con excepciones puntuales y justificadas. En la tabla 3 se detallan las características de dichos cursos, recogiendo el título, el año en que se llevaron a cabo, la duración en horas, los temas tratados y la metodología docente empleada.

Entre el 2014 y el 2016, tres matronas recibieron un total de 16,5 horas de FC sobre VG, y las otras nueve, 22 horas. Los temas de tales cursos, que destacan en las entrevistas, fueron la detección y el abordaje de la VG desde la consulta sanitaria, haciendo especial énfasis en la asistencia a las adolescentes. Asimismo, se trató el abordaje a la violencia sexual y, de forma específica, la violencia hacia los niños y la VG durante el embarazo. La metodología docente predominante fue las clases teóricas. 
Tabla 3. Características de la formación continuada sobre VG realizada por las matronas

\begin{tabular}{|c|c|c|c|c|}
\hline Título & Año & $\begin{array}{l}\text { Número } \\
\text { de horas }\end{array}$ & Temas & $\begin{array}{l}\text { Metodología } \\
\text { docente }\end{array}$ \\
\hline $\begin{array}{l}\text { Curso: Detección y } \\
\text { prevención de la } \\
\text { violencia machista }\end{array}$ & 2014 & 5.5 & $\begin{array}{l}\text { Detección de la VG } \\
\text { El ciclo de la VG } \\
\text { Abordaje de la VG }\end{array}$ & Clase teórica \\
\hline $\begin{array}{l}\text { Jornada: Aproximación a } \\
\text { la VG }\end{array}$ & 2015 & 5.5 & $\begin{array}{l}\text { Detección de la VG } \\
\text { Circuitos de atención } \\
\text { Violencia en los niños } \\
\text { Abusos sexuales } \\
\text { VG en adolescentes } \\
\text { VG en el embarazo }\end{array}$ & Clase teórica \\
\hline $\begin{array}{l}\text { Curso: Sensibilización y } \\
\text { prevención de la VG }\end{array}$ & 2016 & 11 & $\begin{array}{l}\text { VG en los adolescentes } \\
\text { Diversidad sexual y de } \\
\text { género }\end{array}$ & $\begin{array}{l}\text { Taller de } \\
\text { capacitación y } \\
\text { clase teórica }\end{array}$ \\
\hline
\end{tabular}

De los tres cursos mencionados, las matronas destacan elementos que resultaron reveladores y que les han hecho cambiar su percepción de la VG. Como muestra de ello, destaca la percepción de cómo están normalizadas socialmente determinadas formas de violencia hacia las mujeres:

Me encantó porque incluso a veces nosotras mismas no somos conscientes de la violencia que puede haber, porque hay cosas que ya están como estipuladas en la sociedad. (e6)

Por otro lado, una matrona explica que, tras uno de los cursos, una compañera, también profesional sanitaria, le reveló que al asistir se había dado cuenta que ella misma había sufrido VG, y que no fue consciente de ello hasta ese momento, debido esa normalización mencionada.

Las entrevistadas destacan elementos de dichos cursos que les han hecho mejorar sus competencias en el desarrollo de la asistencia clínica, en especial en lo concerniente a la prevención de la homofobia en los adolescentes, como otra forma de violencia relacionada con el género:

Bueno, me fue muy bien de cara a la educación sanitaria con los adolescentes sobre VG [...], es decir que hay que hablar de todas las posibles parejas habidas y por haber, no solo de un chico y una chica. (e4) 
A continuación, se ofrecen las reflexiones de las matronas en relación con sus procesos formativos combinados con los años de práctica profesional.

\section{Capacidad de detectar situaciones de violencia en las mujeres que asisten}

Desde la formación recibida y gracias a su saber experiencial tras años de ejercicio profesional, las matronas identifican diferentes formas de violencia, nombrando principalmente la violencia psicológica, la física y la sexual:

En nuestra sociedad, la violencia física sí que está mal vista, pero otras formas como la sexual está más normalizada [...]. En el postparto me he encontrado a mujeres que dicen que se siente presionadas por sus parejas a reiniciar las relaciones sexuales. (e10)

Opinan que la violencia psicológica es la más frecuente y muy prevalente en las mujeres que asisten, aunque opinan que posiblemente está infradetectada:

Yo creo que es mucho más frecuente de lo que nosotros detectamos. Yo puedo decir que he detectado en todo el tiempo que llevo de matrona dos casos de violencia en el embarazo, y seguro que he estado delante de muchos más. (e10)

Consideran que su capacidad en la detección de situaciones de violencia se rige por su percepción y sensibilidad. En general, no preguntan de forma sistemática sobre VG. Sin embargo, explican que sí lo hacen si detectan algún signo de sospecha, ya sea físico o psicólogo:

Me hace sospechar que no se presente a las visitas, que llegue tarde, ves algo y sí empiezas a indagar [...]. Entonces intento quedarme a solas con la mujer.

Algunas matronas expresan que no preguntan de forma directa sobre VG, sino que tienen presente la posibilidad y lo preguntan de modo indirecto al interesarse por el estado emocional de la mujer y la relación con su pareja:

Yo lo que hago como de una forma natural es preguntar: ¿está contenta?, ¿tu pareja te ayuda? [...]. Lo hago de esta manera, ¿qué tal?, y ¿qué dice tu pareja?... (e6) 
También alertan que cada vez detectan más casos en las adolescentes que asisten, principalmente violencia psicológica y sexual. Por ejemplo, cuando las chicas a las que asisten manifiestan que se sienten presionadas, de una u otra manera, a tener relaciones sexuales sin protección:

Visitamos muchas chicas jovencitas que vienen por la pastilla postcoital o vienen para información de interrupción voluntaria del embarazo y te dicen que él se ha negado a ponerse el preservativo. (e1)

Sin embargo, entre las entrevistadas, existen dudas respecto a cuáles son los límites para considerar que una mujer se encuentra o no bajo una situación de violencia. En la siguiente transcripción se ofrece una ejemplificación de tales dudas:

Las mujeres te dicen: el preservativo no se lo pone, antes cambia de pareja [...]. Yo a esto ya me he acostumbrado, me parece fatal, pero no diría que es violencia. (e3)

\section{Conocimientos sobre el perfil de las víctimas}

Las matronas hacen notar que, a su entender, cualquier mujer puede ser víctima de VG independientemente de su nivel sociocultural o económico, y que no hay un perfil concreto. Aunque algunas sí aluden a la posibilidad de que exista una mayor prevalencia de casos en las mujeres que han vivido situaciones de VG en la infancia o que han sido educadas en sociedades intensamente patriarcales que normalizan ciertas formas de VG:

Pero sí que quizás más frecuentemente en mujeres que desde pequeñas han tenido una educación de que el hombre es superior, que hayan podido vivir otros casos [...] pueden vivir la violencia como normal o incluso merecida. (e11)

Algunas matronas consideran que las embarazadas o las mujeres que acaban de tener un bebé pueden ser más susceptibles de sufrir violencia, principalmente ocasionado por los cambios de rol en la pareja. También consideran que a determinadas mujeres que asisten les resultará más difícil salir de esa situación violenta, a causa de su falta de independencia económica o la existencia de uno o varios hijos. Primordialmente las mujeres inmigrantes, a las que se añade, en muchas ocasiones, que desconocen el idioma de la sociedad de llegada y los recursos sociales disponibles a tal efecto: 
Aquí están a lo mejor solo con su pareja o con su pareja y sus hijos, dicen: ¿dónde voy?, cuando además muchas veces hay una dificultad idiomática añadida. (e10)

\section{Conocimientos sobre el perfil de los agresores}

A partir de su formación y su experiencia profesional afirman que los hombres maltratadores no cumplen tampoco un único perfil sociocultural o económico. Pueden ser hombres que se comportan correctamente en la consulta cuando acompañan a sus parejas, sin generar sospechas de su carácter violento en el ámbito familiar:

No creo que haya un perfil concreto. Puede ser un hombre amable, educado, que nunca te lo imaginarías. (e7)

\section{Conocimientos en relación con las repercusiones de la VG en la salud de las mujeres}

Reconocen que la VG puede repercutir gravemente en la salud de la mujer, tanto en el ámbito físico como psicológico. Sugieren que, en el caso de las embarazadas, los daños se amplían al poder afectar al feto, al curso del embarazo, al parto y al cuidado del recién nacido, como podemos observar en las siguientes afirmaciones:

El estado de nerviosismo constante de la mujer, que tenga miedo, le tiene que afectar de alguna forma, esa tensión se puede transmitir al feto. (e11)

\section{Capacidad en el abordaje de los casos}

Para el abordaje de los casos, se basan, fundamentalmente, en su criterio individual, fundamentado en la formación recibida y la experiencia acumulada. Aunque saben que existen protocolos oficiales, consideran que no los usan tanto como deberían. Explican que no reciben información o formación específica cada vez que se modifica o se incluye un nuevo protocolo o documento en el web del Departamento de Salud. Por otra parte, la presión asistencial no les permite tampoco poner al día sus conocimientos. Las matronas explican que deben hacerlo fuera del horario laboral y por propia iniciativa. Todo ello influye en la percepción generalizada de que sus conocimientos al respecto no están suficientemente actualizados:

Si hay protocolos, pero nosotros no sabemos que existen... en intranet [Red informática interna de la institución sanitaria] estarán, pero no puedo estar mirando cada día los protocolos que hay, no tengo tiempo. (e11) 
Todas las entrevistadas señalan que, ante la detección de un caso de violencia, actúan derivando a la mujer a otros profesionales. Derivan a los servicios sociales del propio centro sanitario o les facilitan a las mujeres el número de teléfono de atención a las víctimas (disponible para todo el país). Solo tres nombran los centros con servicio de intervención especializada (SIE) disponibles en el municipio para las víctimas de VG:

También los centros SIE que hacen un abordaje multidisciplinar. Pero yo trabajo mucho con los servicios sociales del ambulatorio, porque puedes hablar con ellos personalmente. (e10)

\section{Necesidades formativas}

Se recogen a continuación, cuáles son las demandas, necesidades e intereses de las matronas en este tema, así como las orientaciones generales de cara a su satisfacción.

\section{Desarrollar la formación}

Las matronas señalan que les gustaría profundizar más en su formación a través de cursos específicos que tengan contenidos tales como las manifestaciones de la VG en las diferentes culturas y pautas para abordarlas. El alto nivel de diversidad cultural entre las mujeres que asisten incrementa la dificultad y complejidad de la detección. Prefieren cursos dirigidos y programados específicamente para ellas, puesto que podrían dar respuesta a circunstancias concretas con las que se encuentran en su ejercicio profesional. Se muestran interesadas, receptivas y optimistas para seguir formándose en este tema y mejorar así la asistencia.

\section{Formación para la detección}

Demandan mayor formación para abordar la entrevista con la mujer, es decir, saber cómo preguntar y mostrarse receptivas y sensibles para crear un clima de intimidad que favorezca que la mujer se sienta cómoda para explicar su situación. Además, necesitan estrategias que les permitan hacerlo a pesar de la presión del tiempo limitado que tienen para cada consulta:

Aprender a trabajar la confianza para poder llegar a que la mujer se sincere.

[...] Echo de menos formación a nivel general en estos temas para que seamos sensibles y seamos sensibles a la hora de abordarlos. La mujer tiene que tener la confianza y que tú sepas de qué manera abordarla. (e11) 
Por otro lado, conceden una gran importancia a saber observar, acostumbrarse a ello y reconocer indicadores indirectos de riesgo, aquellos que advierten de la posibilidad de que una mujer esté sufriendo VG.

Tendríamos que tener la misma habilidad para detectar violencia que la que tenemos para detectar si hay algún problema físico [...]. Esto es lo que nos falta, saber cuándo tenemos que estar alerta. (e8)

También indican que sería útil poder aplicar cuestionarios estandarizados que aportaran mayor objetividad en la detección, así como estar entrenadas y habituadas a su uso:

Estaría bien cuando se sospecha algo tener herramientas, no sé quizás tener un cuestionario ya estipulado para hacer determinadas preguntas. (e6)

\section{Capacitación para el abordaje}

Las matronas consideran que es importante conocer de forma más detallada los circuitos de derivación a los que dirigir a las mujeres, pero dan más importancia a saber acoger emocionalmente a las mujeres víctimas de VG y asesorarlas una vez que han revelado su situación. En el mismo sentido, piensan que es preciso un mayor y más detallado conocimiento de los recursos disponibles que tendrá la mujer en caso de querer denunciar la situación, en referencia a ayudas sociales, económicas y judiciales, así como los procedimientos concretos que tendrá que seguir. Creen que informar a las mujeres de esto les daría seguridad.

Destacan que necesitan conocer mejor esos procedimientos, también para protegerse como profesionales. En relación con ello, les preocupan las cuestiones éticas, no traspasar los límites del respeto a la mujer y a sus decisiones. Así, por ejemplo, mencionan la necesidad de conocer la forma más conveniente de registrar en la historia clínica los casos de VG por respeto a la privacidad de la mujer:

El registro en la historia clínica me crea un poco de ambigüedad, porque no sé si estoy inmiscuyéndome en la intimidad de alguien, sobre todo cuando no quieren denunciar [...]. Yo no sé aquí cómo lo tendríamos que hacer, también a nivel jurídico. (e6) 


\section{Currículum formativo de los formadores}

Consideran necesario que se desarrolle una formación holística, es decir, que las capacite para atender a la mujer en toda su complejidad. Así proponen contenidos aportados desde otros ámbitos además del sanitario: jurídico, psicológico, sociológico y antropológico. Demandan que los docentes les puedan aportar no solamente conocimientos teóricos, sino, sobre todo, experiencia profesional desde su tarea asistencial en servicios directamente relacionados con la VG. De esta forma pueden compartir con ellas su experiencia:

Gente que esté muy involucrada en ayudar a estas mujeres, que lo vea cada día [...], porque esa gente sabrá cómo podemos detectarlo, tienen mucha información [...], yo creo que son la gente que más nos pueden abrir los ojos. (e8)

\section{Propuestas relacionadas con la metodología docente}

Las matronas demandan metodologías activas. Explican que les gustaría profundizar en análisis de casos. También mencionan el role-playing como técnica para poner en práctica algunas habilidades y confrontar alternativas. Proponen debates, visualización de vídeos o incluso la escucha de testimonios. Plantean que les gustaría que se invitara a los cursos de FC a alguna mujer que haya sufrido VG y quiera compartir su vivencia.

Incluso estaría bien que viniera una persona que ha sufrido VG [...] porque estos casos calan en la gente. (e8)

\section{DISCUSIÓN}

A diferencia de otros trabajos (Yildiz, Selimen, y Dogan, 2014), todas las matronas entrevistadas en este estudio tenían formación sobre VG. La mayoría de esta formación había sido recibida como formación continuada (FC), impartida por la propia institución en la que trabajan y en horario laboral. Solo una minoría recuerdan haberlo tratado en los estudios universitarios, lo cual coindice con los resultados de Crombie et al. (2016). Las matronas recibieron un total de entre 16,5 y 22 horas de FC, lo cual supera lo revelado por otros estudios en los que las matronas habían recibido cursos de formación de una media de 7,5 horas (Salmon et al., 2006) o incluso solamente 3 horas (Protheroe, Green, y Spiby, 2004). Los contenidos recibidos en la FC se centraron fundamentalmente en la 
detección y el abordaje de la VG, el ciclo de la violencia y los circuitos de atención. Esto es semejante a los resultados de otros trabajados (Crombie et al., 2016) donde en la formación recibida por las matronas se abordaba cómo llevar a cabo el cribado y la planificación para ayudar a la mujer a salir de la situación de violencia. Por otro lado, las matronas entrevistadas recibieron además formación específica sobre violencia sexual, violencia hacia los niños y violencia durante el embarazo y la adolescencia. La metodología docente predominante fue la clase teórica de tipo expositivo, igual que los resultados de otros autores (Protheroe, Green y Spiby, 2004). Posiblemente justificada por las pocas horas de formación en relación con la complejidad de la VG y la necesidad de un enfoque multidisciplinar. Coincidiendo con otros hallazgos (Baird et al., 2015), todas las entrevistadas tenían un fuerte compromiso personal con la VG. Se muestran receptivas y optimistas para seguir formándose en este tema y mejorar la asistencia. Conocen que las situaciones de VG durante el embarazo repercuten en la salud de las mujeres y sus hijos, y consideran que posiblemente esté infradetectada.

Tienen conocimientos teóricos correctos sobre la VG, pero al igual que en otros trabajos (Bradbury-Jones y Broadhurst, 2015; Eustace et al., 2016), no se consideraban suficientemente capacitadas. Mauri et al. (2015) revelan que las matronas no se sentían preparadas para reconocer los signos de violencia y no sabrían cómo manejar la situación si tuvieran que hacerlo, lo cual es similar a lo manifestado por las matronas entrevistadas y muestra la necesidad de trabajar en la mejora de esa percepción de seguridad. Las matronas demandan más formación, primordialmente en cuestiones íntimamente relacionadas con la práctica diaria: detección de los casos, técnicas de entrevista, uso de cuestionarios estandarizados, reconocimientos de signos de maltrato, información de los recursos disponibles y los circuitos de derivación al detectar un caso (Di Giacomo, et al. 2017).

Manifiestan que les gustaría estar mejor capacitadas para conseguir crear una relación de confianza con las mujeres, puesto que consideran que su actitud es fundamental para que las víctimas revelen su situación. Esto es semejante a los resultados de otros trabajos (Bradbury-Jones, Clark, Parry, y Taylor, 2017), reforzando nuevamente la idea de una preparación que incluya también recursos psicológicos de relación con las mujeres. Proponen que en futuros cursos de FC el enfoque sea pluridisciplinar, y que los formadores provengan de ámbitos profesionales donde se trabaje con mujeres víctimas de VG. Opinan que las metodologías docentes más enriquecedoras para tratar estos temas 
son las activas, realizadas en grupos reducidos de alumnos, con inclusión de análisis de casos, escucha de testimonios o los role-playing. Todas estas metodologías docentes han sido empleadas por otros autores para formar sobre VG a enfermeros, médicos y matronas con resultados positivos en el aprendizaje (véase, por ejemplo: Baird et al., 2018), dado que permiten desarrollar procesos no solo cognitivos, sino también afectivos y con ello conseguir aprendizajes más significativos. Cabe señalar como limitación de estudio que, al ser cualitativo centrado en el contexto de las matronas de AP en la población de L'H, los resultados solo pueden ser transferibles a otros contextos con características semejantes.

\section{CONCLUSIONES}

Las matronas se muestran sensibilizadas, todas ellas tienen formación adquirida de manera formal, mayoritariamente desde su propio centro de trabajo, pero su capacitación es también fruto de su prolongado saber experiencial derivado de los años de ejercicio profesional. No obstante, y a pesar de la formación recibida, reclaman incrementar su capacitación mediante un programa sólido de FC que les permita mejorar sus capacidades en cuanto a saber formular las preguntas apropiadas, reconocimiento de señales de VG, instrumentos comunes de detección, recursos para gestionar posibles obstáculos y clarificación respecto al circuito de derivación de las mujeres en caso de que revelen una situación de violencia, a partir de los protocolos y planes de actuación existentes.

También demandan que les aporte herramientas para mejorar la escucha activa y las habilidades de acogida que favorezcan la creación de un clima de confianza, absolutamente necesario para que la mujer revele su situación. Proponen incluir metodologías activas, con docentes con experiencia profesional en la asistencia a víctimas de VG, provenientes de diferentes disciplinas con el objetivo de abordar el tema desde todas sus perspectivas: sanitaria, psicológica, social, ética y legal. Parece conveniente atender a estos intereses formativos como una vía para contribuir a erradicar la VG desde el ámbito sanitario.

\section{AGRADECIMIENTOS}

Las autoras de este artículo queremos dar las gracias a las matronas que han compartido con nosotras sus experiencias, conocimientos y reflexiones. 


\section{BIBLIOGRAFÍA}

BAIRD, K., et al. An exploration of Australian midwives' knowledge of intimate partner violence against women during pregnancy. En: Women and Birth. 2015, vol. 28, núm. 3, pp. 215-220.

BAIRD, K., et al. Effectiveness of training to promote routine enquiry for domestic violence by midwives and nurses: A pre-post evaluation study. En: Women and Birth. 2018, vol. 31, núm. 4, pp. 285-291.

BradBuRY-Jones, C.; Broadhurst, K. Are we failing to prepare nursing and midwifery students to deal with domestic abuse? Findings from a qualitative study. En: Journal of Advanced Nursing. 2015, vol. 71, núm. 9, pp. 2062-2073.

BRADBURY-JONES, C., et al. Development of a practice framework for improving nurses' responses to intimate partner violence. En: Journal of Clinical Nursing. 2017, vol. 26, núm. 15, pp. 2495-2502.

Crombie, N.; Hooker, L.; \& Reisenhofer, S. Nurse and midwifery education and intimate partner violence: a scoping review. En: Journal of Clinical Nursing. 2016, vol. 26, núm. 15, pp. 2100-2125.

Delegación del Gobierno para la Violencia de GÉnERo. Macroencuesta de violencia contra la mujer 2015. Gobierno de España, 2015. Disponible en: www.violenciagenero.igualdad.mpr.gob.es/violenciaEnCifras/estudios/colecciones/ pdf/Libro_22_Macroencuesta2015.pdf

Di Giacomo, P., et al. Violence against women: knowledge, attitudes and beliefs of nurses and midwives. En: Journal of Clinical Nursing. 2017, vol. 26, núm. 15-16, pp. 2307-2316.

EUSTACE, J., et al. Midwives' experiences of routine enquiry for intimate partner violence in pregnancy. En: Women and Birth. 2016, vol. 29, núm. 6, pp. 503-510.

Generalitat de Catalunya. Institut d'Estadística de Cataluña. GC, 2017. Disponible en: www.idescat.cat

Generalitat de Cataluña. Cartera de Serveis de les Unitats d'Atenció a la Salut Sexual i Reproductiva de Suport a l'Atenció Primària. GC, 2017. Disponible en: https://salutweb.gencat.cat/web/.content/_ambits-actuacio/Linies-dactuacio/Serveis$\underline{\text { sanitaris/Salut-sexual-reproductiva/Model_cartera_de_serveis/placarte.pdf }}$

Gómez-Fernández, M. A., Goberna-Tricas, J., \& PayÀ-SÁnchez, M. Intimate partner violence as a subject of study during the training of nurses and midwives in 
Catalonia (Spain): A qualitative study. En: Nurse Education in Practice. 2017, vol. 27, pp. 13-27.

GrANeHEIM, U. H.; LundMAN, B. Qualitative content analysis in nursing research: Concepts, procedures and measures to achieve trustworthiness. En: Nurse Education Today. 2004, vol. 24, núm. 2, pp. 105-112.

James, L.; Brody, D.; Hamilton, Z. Risk factors for domestic violence during pregnancy: a meta-analytic review. En: Violence \& Victims. 2013, vol. 28, núm. 3, pp. 359-380.

Jefatura Del Estado. Ley Orgánica 1/2004, de 28 de diciembre, de Medidas de Protección Integral contra la Violencia de Género. En: Boletín Oficial del Estado. Gobierno de España, 2004.

MACKEY, S. Phenomenological nursingresearch: methodological insights derived from Heidegger's interpretive phenomenology. En: International Journal of Nursing Studies. International Journal of Nursing Studies. 2005, vol. 42, núm. 2, pp. 179-186.

MAURI, E., et al. Domestic violence during pregnancy: Midwives' experiences. En: Midwifery. 2015, vol. 31, núm. 5, pp. 498-504.

NACIONES UnidAS. Declaración sobre la eliminación de la violencia contra la mujer. Resolución de la Asamblea General. Naciones Unidas, 1993. Disponible en: www.aulaviolenciadegeneroenlocal.es/consejosescolares/archivos/declaracion_sobr e_la_eliminacion_de_la_violencia_contra_la_mujer.pdf

NACIONES UNIDAS. Supplement to the handbook on violence for legislation against women. Naciones Unidas, 2011. Disponible en: www.un.org/womenwatch/daw/vaw/v-handbook.htm

ORGANIZACIÓN MUNDIAL DE LA SALUD. Intimate partner violence during pregnancy. OMS, 2011. Disponible en:

apps.who.int/iris/bitstream/handle/10665/70764/WHO_RHR_11.35_eng.pdf?seque $\underline{\text { nce }=1}$

ORgANIZACIÓN MUNDIAL DE LA SALUD. Global and regional estimates of violence against women: prevalence and health effects of intimate partner violence and nonpartner sexual violence. OMS, 2013. Disponible en: www.who.int/reproductivehealth/publications/violence/

ORgANIZACIÓN MUNDIAL DE LA SALUD. Violence against women. Intimate partner and sexual violence against women. OMS, 2017. Disponible en: www.who.int/mediacentre/factsheets/fs239/en/ 
Protheroe, L.; Green, J.; Spiby, H. An interview study of the impact of domestic violence training on midwives. En: Midwifery. 2004, vol. 20, núm. 1, pp. 94-103.

SAlmon, D., et al. An evaluation of the effectiveness of an educational programme promoting the introduction of routine antenatal enquiry for domestic violence. Midwifery. 2006, vol. 22, núm. 1, pp. 6-14.

Skrtic, T. M. Doing Naturalistic Research into Educational Organisations. En: Lincoln, Yvonna S. (ed.), Organizational theory and inquiry: the paradigm revolution. London: SAGE, 1985.

TAYLOR, S.; BOGDAN, R. Introducción a los métodos cualitativos de investigación. La búsqueda de significados. Barcelona: Paidós, 1987.

Yildiz, T.; Selimen, D.; Dogan, D. A Study Comparing the Pre- and Post-Training Knowledge of Emergency Department Nurses in Turkey for the Diagnosis of Physically Abused Women. En: Journal of Family Violence. 2014, vol. 29, núm. 5, pp. 519-525. 